\title{
Influence of the Static Dynamic Ergodic Divertor on Edge Turbulence Properties in TEXTOR
}

\author{
Y. Xu, ${ }^{1}$ R. R. Weynants, ${ }^{1}$ S. Jachmich, ${ }^{1}$ M. Van Schoor, ${ }^{1}$ M. Vergote, ${ }^{1}$ P. Peleman, ${ }^{2}$ M. W. Jakubowski, ${ }^{3}$ M. Mitri, ${ }^{3}$ \\ D. Reiser, ${ }^{3}$ B. Unterberg, ${ }^{3}$ K. H. Finken, ${ }^{3}$ and the TEXTOR team \\ ${ }^{1}$ Laboratoire de Physique des Plasmas-Laboratorium voor Plasmafysica, \\ Association "Euratom-Belgian State," Ecole Royale Militaire-Koninklijke Militaire School, B-1000 Brussels, Belgium* \\ ${ }^{2}$ Department of Applied Physics, Ghent University, B-9000 Ghent, Belgium \\ ${ }^{3}$ Institute für Plasmaphysik, Forschungszentrum Jülich, D-52425 Jülich, Germany*
}

(Received 11 May 2006; published 19 October 2006)

\begin{abstract}
Systematic measurements on the edge turbulence and turbulent transport have been made by Langmuir probe arrays on TEXTOR under various static Dynamic Ergodic Divertor (DED) configurations. Common features are observed. With the DED, in the ergodic zone the local turbulent flux reverses sign from radially outwards to inwards. The turbulence properties are profoundly modified by energy redistribution in frequency spectra and suppression of large scale eddies. The fluctuation poloidal phase velocity changes direction from electron to ion diamagnetic drift, consistent with the observed reversal of the $E_{r} \times B$ flow. In the laminar region, the turbulence is found to react to an observed reduced flow shear.
\end{abstract}

DOI: 10.1103/PhysRevLett.97.165003

PACS numbers: 52.55.Fa, 52.25.Fi, 52.35.Ra, 52.35.Vd

One of the present challenges in the fusion reactor operation is the control of the edge plasma parameters. Results on the tokamaks TEXT [1], Tore Supra [2], DIII-D [3], and TEXTOR [4] have demonstrated that an ergodized magnetic boundary can be effective to optimize the plasma-wall interaction. Meanwhile, the local effects of the magnetic ergodization on edge turbulence and turbulent cross-field transport have also been studied both experimentally $[1,5,6]$ and theoretically $[7,8]$. It has been observed on TEXT [1] and Tore Supra [2,5,6] that in the ergodic divertor (ED) configuration the edge density fluctuations are decreased whereas the turbulent cross-field diffusivity is less affected. However, a systematic investigation of the turbulence properties, such as frequency and wave-number spectra and the fluctuation propagations, has not been made or was done for a reduced set of wavenumber values [5]. A distinct description in the ergodic and laminar zones was also not given.

Recently, on the tokamak TEXTOR the Dynamic Ergodic Divertor (DED) [4] has been installed at the high-field side of the torus $(R / a=1.75 / 0.47 \mathrm{~m})$, contrary to other machines where the ED coils were mounted at the low-field side [1-3]. The DED consists of 16 perturbation coils oriented parallel to the field lines on the magnetic flux surface with a safety factor $q \cong 3$. With different current distributions in the coils, the base poloidal/toroidal modes, $m / n$, can be adjusted as $12 / 4,6 / 2$, and $3 / 1$. The penetration depth into the plasma depends on $m$ : In 12/4 the influence is restricted to the plasma boundary, while in $3 / 1$ it can reach much deeper. In the outer plasma layer, DED induces stochastization of the magnetic field lines, including an ergodic zone with long and a laminar zone with short connection lengths to the wall [9]. In this Letter, we present the first systematic measurements by Langmuir probe arrays on the edge turbulence properties and fluctuation-driven transport in the presence of various static DED configurations (dc current on the coils).

To get effective impacts of the DED at the plasma boundary, the discharge conditions have been optimized as follows: For $m / n=12 / 4, I_{p}=250 \mathrm{kA}, B_{T}=1.4 \mathrm{~T}$, $R / a \cong 1.73 / 0.46 \mathrm{~m}$, dc DED current $I_{\mathrm{DED}}=12 \mathrm{kA}$; for $6 / 2, \quad I_{p}=270 \mathrm{kA}, \quad B_{T}=1.9 \mathrm{~T}, \quad R / a \cong 1.73 / 0.46 \mathrm{~m}$, $I_{\mathrm{DED}}=6 \mathrm{kA}$; and for $3 / 1, I_{p}=250 \mathrm{kA}, B_{T}=1.9 \mathrm{~T}$, $R / a \cong 1.75 / 0.48 \mathrm{~m}, I_{\mathrm{DED}}=1 \mathrm{kA}$. The $I_{\mathrm{DED}}$ is applied during the stationary phase of the Ohmic discharge. In all cases, no external tearing modes are excited, the lineaveraged plasma density is $(1.5-2.0) \times 10^{19} \mathrm{~m}^{-3}$, and the global confinement is not affected. To measure the edge plasma parameters and their fluctuations, two Langmuir probe arrays were installed at the outer midplane (low-field side) at two different toroidal locations. One array is stationary while the other is fast reciprocating. The probe measurements cover both the ergodic and the laminar zones. The results from the two probe systems are quite similar, independent of the toroidal locations. The turbulence properties also display similarity for the different DED scenarios.

In this study, the local electron temperature $T_{e}$, density $n_{e}$, and floating potential $\phi_{f}$ were measured by a triple probe [10] assembled in each of the probe systems. The radial electric field $E_{r}$ is derived from the plasma potential $\phi_{p}=\phi_{f}+2.5 T_{e}$ [11]. The turbulent particle flux $\Gamma_{\mathrm{fl}}$ is computed from the fluctuating density $\left(\tilde{n}_{e}\right)$ and poloidal electric field $\left(\tilde{E}_{\theta}\right)$ by $\Gamma_{\mathrm{fl}}=\left\langle\tilde{n}_{e} \tilde{E}_{\theta}\right\rangle / B_{\phi}$, where $\langle\cdots\rangle$ denotes an ensemble average. Here, $\tilde{n}_{e}$ is estimated in the usual way from the ion saturation current fluctuations $\tilde{I}_{s}$ detected by the triple probe and $\tilde{E}_{\theta}$ is estimated from two poloidally spaced measurements of floating potential fluctuations $\tilde{\phi}_{f}$. The fluctuation data were sampled at $500 \mathrm{kHz}$. Using the standard two-point cross-correlation technique 
[12], the poloidal (radial) fluctuation spectra $S(k, f)$, power-averaged dispersion relation $\bar{k}(f)$, and $k$ spectrum width were obtained by analyzing the fluctuation data collected on two poloidally (radially) separated probe pins.

Figure 1 shows the typical radial dependence of the edge equilibrium parameters before (black curves) and during (red or gray curves) the static DED phases measured in $m / n=6 / 2$ DED by the fast reciprocating probe. In the figure, the vertical solid line denotes the limiter position. The dashed line roughly separates the ergodic zone (EZ, left side) and the laminar zone (LZ, right side), the distinction being made by the connection length of field lines, $L_{c}$, which is about 3-4 poloidal turns at the position of the dashed line as predicted by the calculations [9]. As such, the $L_{c}$ in the EZ is longer than the Kolmogorov length, whereas in the LZ it is shorter. The Chirikov parameter is larger than 1 in the EZ. From Fig. 1(a), we can see that with DED the local electron temperature $T_{e}$ is strongly reduced in the LZ and in the scrape-off layer defined by the limiter. Inside EZ, the change in $T_{e}$ is much less pronounced. Similar reduction of $T_{e}$ in the ED configuration was also observed in Tore Supra [2]. Figure 1(b) shows that the plasma density increases and decreases, respectively, inside and outside the limiter position during the DED. The local pressure $P_{e}\left(=n_{e} T_{e}\right)$ gradient is hence slightly enhanced across the perturbation region, as seen in Fig. 1(c). During DED, the edge floating potential $\phi_{f}$ is always
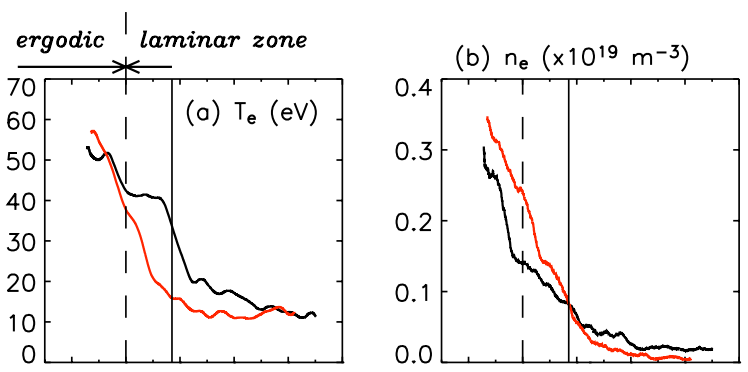

(c) $\mathrm{P}_{\mathrm{e}}\left(\times 10^{19} \mathrm{~m}^{-3} * \mathrm{eV}\right)$
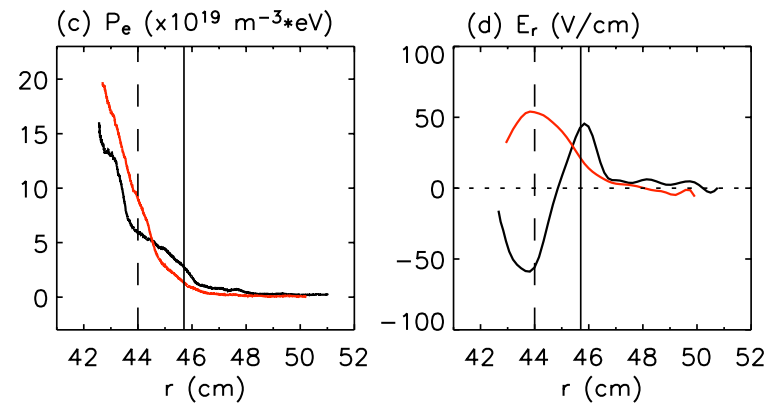

FIG. 1 (color online). Radial profiles of the edge plasma parameters before (black curves) and during (red or gray curves) the static DED measured by fast reciprocating Langmuir probe arrays in an $m / n=6 / 2$ DED discharge on TEXTOR (No. 99626). (a) Electron temperature; (b) plasma density; (c) electron pressure; (d) radial electric field. The vertical solid line indicates the limiter position $(a \approx 45.7 \mathrm{~cm})$. The vertical dashed line roughly separates the ergodic (left side) and the laminar (right side) zones. increased to zero or positive from the negative values before DED, leading to a change of the $E_{r}$ sign from negative to positive in most of the LZ and EZ area [see Fig. 1(d)]. The $E_{r} \times B$ shear rate is, however, reduced by the DED at all measured radial locations. Similar changes in $E_{r}$ were observed earlier on TEXT [13] and recently on TEXTOR by other diagnostics [14]. A possible explanation is that with ED the electrons move faster than massive ions along the field lines to the wall so that a positive $E_{r}$ must be created to restore the ambipolarity. Theory [1517] predicts that the negative neoclassical ambipolar electric field will be reduced when the radial conductivity associated with the magnetic field stochasticty $\sigma_{\text {st }}$ becomes of the order of the neoclassical conductivity $\sigma_{\text {neo }}$. The first of these is given by $\sigma_{\text {st }} \approx O\left(\sigma_{\|} b_{r}^{2}\right)$, where $\sigma_{\|}$is the Spitzer-Harm conductivity, of order $10^{6}$, and $b_{r}$ is the ratio of the perturbed to the main magnetic field. In TEXTOR, $\sigma_{\text {neo }}$ is measured by biasing [18] and reaches under the present conditions about $10^{-3} \Omega^{-1} \mathrm{~m}^{-1}$. The here applied DED perturbation $b_{r}\left(O_{10}^{-4}\right)$ is therefore more than adequate to bring about the observed $E_{r}$ changes. To illustrate the impact of the static DED on edge fluctuations and turbulent transport, Fig. 2 displays time traces of $I_{\mathrm{DED}}$, fluctuation power of $\tilde{n}_{e}$ and $\tilde{E}_{\theta}$, and the ensemble-averaged turbulent flux $\left\langle\Gamma_{\mathrm{fl}}\right\rangle$, measured in $m / n=12 / 4$ DED by a stationary probe array located inside the ergodic layer. Figures 2(a)-2(c) indicate that during DED the fluctuation amplitudes in $\tilde{E}_{\theta}$ are reduced considerably while for $\tilde{n}_{e}$ the modification is small. In Fig. 2(d), it is interesting to see that with DED the $\left\langle\Gamma_{\mathrm{fl}}\right\rangle$ changes remarkably and even

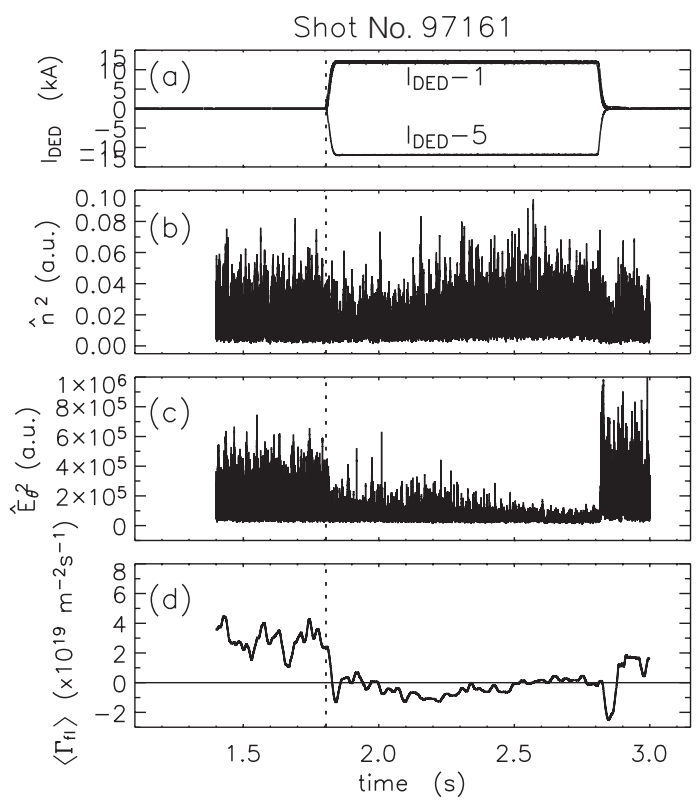

FIG. 2. Time traces of (a) DED currents in coil 1 (thick line) and coil 5 (thin line); (b) power of density fluctuations; (c) power of $\tilde{E}_{\theta}$ fluctuations; and (d) ensemble-average of fluctuationdriven particle flux, $\left\langle\Gamma_{\mathrm{fl}}\right\rangle$, measured by a stationary triple probe in the ergodic region $(r \approx 43 \mathrm{~cm})$ of $m / n=12 / 4$ static DED. The dotted lines denote the start time of DED. 
reverses sign from radially outwards $(>0)$ to inwards $(<0)$, on account of a change in the phase shift between $\tilde{n}_{e}$ and $\tilde{E}_{\theta}$. The inward flux during DED is consistent with the slight increase of the local pressure gradient in the stochastic region [see Fig. 1(c)]. Plotted in Figs. 3(a) and 3(b) are the radial profiles of $\left\langle\Gamma_{\mathrm{fl}}\right\rangle$ measured by the fast probes in the $6 / 2$ and $3 / 1$ static DED. It is noted that before DED (crosses), $\left\langle\Gamma_{\mathrm{fl}}\right\rangle$ in either case exhibits a reduction inside the limiter location, which might be attributed to the local decorrelation role of the sheared $E_{r}$ [see Fig. 1(d)], as observed earlier in other machines [19]. In Fig. 3, we can clearly see that with DED the $\left\langle\Gamma_{\mathrm{fl}}\right\rangle$ both change direction in the ergodic region. The prevailing $E_{r} \times$ $B$ flow velocity, $V_{E \times B}=E_{r} / B_{\phi}$, can be inferred from Fig. 1(d) and apparently the local flow shear modifications cannot account for the observed flux changes. As the global confinement is not affected, a compensation of outflux should arise either from the fluctuation-driven flux at other locations or from the ergodic particle transport across the stochastic layer [2].

Further analysis of the frequency power spectrum, $S(f)$, of $\tilde{n}_{e}, \tilde{\phi}_{f}$, and $\tilde{E}_{\theta}$ indicate that in the Ohmic discharge before DED there exist coherent modes in all fluctuating fields at the plasma edge with peaks at about 10 and $50 \mathrm{kHz}$, as shown by black curves in Fig. 4(a) for density fluctuations. During the static DED, the modes are all destroyed in the ergodic region and the shape of $S(f)$ becomes exponential (see red or gray curves). These results suggest that the magnetic ergodization causes an energy redistribution process of turbulence at different frequency components. Such effects are less evident in the laminar zone. In Fig. 4(b), the poloidal wave-number spectra, $S\left(k_{\theta}\right)$, of potential fluctuations before (in black) and during (in red or gray) the DED are plotted. Here, $k>0(<0)$ is in the electron (ion) diamagnetic drift direction, $\omega_{*, e}\left(\omega_{*, i}\right)$. Several features can be seen: (i) before DED the $S\left(k_{\theta}\right)$ is power weighted in the $\omega_{*, e}$ direction; (ii) with DED the fluctuation power in the range $-1<k_{\theta}<5 \mathrm{~cm}^{-1}$ is largely reduced, while for $k_{\theta}<-1.3 \mathrm{~cm}^{-1}$ the power is

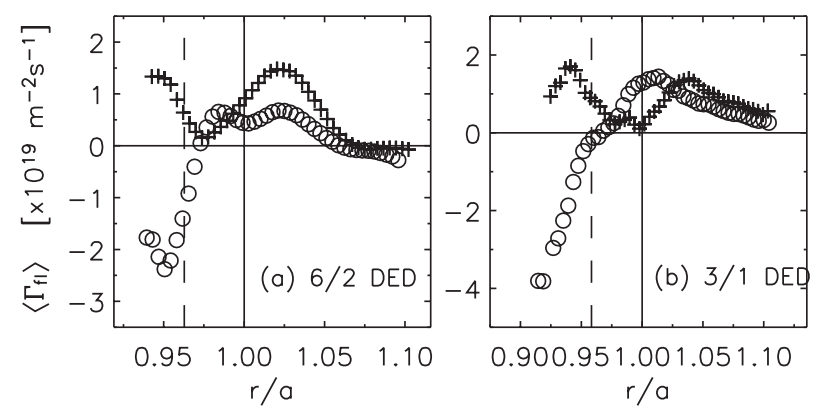

FIG. 3. Radial profiles of fluctuation-driven particle flux $\left\langle\Gamma_{\mathrm{fl}}\right\rangle$ measured by a fast reciprocating triple probe array before (crosses) and during (open circles) the static (a) $m / n=6 / 2$ and (b) $m / n=3 / 1$ DED phases. The radial locations are normalized by $a$. The vertical dashed line separates the ergodic (left side) and laminar (right side) zones. slightly increased, implying a change in the propagation of fluctuations from $\omega_{*, e}$ to $\omega_{*, i}$ direction; (iii) the normalized $S\left(k_{\theta}\right)$ drawn in the inset is flattened during DED, indicating a broadening of the $k_{\theta}$ spectrum and thus a reduction of turbulence correlation length; (iv) the strong reduction of $S\left(k_{\theta}\right)$ at small $k_{\theta}$ reveals the suppression of large structure turbulence by DED, as observed earlier [5]. The influence of DED on turbulent eddies can be further seen from the radial (poloidal) correlation length, $l_{\mathrm{cr}}\left(l_{c \theta}\right)$, which is defined as the inverse of the $k_{r}\left(k_{\theta}\right)$ spectrum width detected by two radially (poloidally) separated probes [12]. Shown in Fig. 5 are the radial profiles of $l_{\text {cr }}$ and $l_{c \theta}$ measured before (circles) and during (triangles) the 6/2 DED. With DED, both $l_{\mathrm{cr}}$ and $l_{c \theta}$ are reduced in the EZ, suggesting a destruction of turbulence eddies by the magnetic ergodization. In contrast, $l_{\mathrm{cr}}$ and $l_{c \theta}$ are both enhanced in the LZ. On account of the much weaker stochastization there, the turbulence appears only to react to the observed reduction of $E_{r} \times B$ flow shear rate in that region [see Fig. 1(d)].

The fluctuation poloidal phase velocity, $V_{\mathrm{ph}}$, is calculated as $V_{\mathrm{ph}}=\sum S\left(k_{\theta}, f\right)\left(2 \pi f / k_{\theta}\right) / \sum S\left(k_{\theta}, f\right)$. Figure 6 shows the radial dependence of $V_{\mathrm{ph}}$ before and during the $6 / 2$ static DED together with the $E_{r} \times B$ velocity, $V_{E \times B}$. It can be seen that either before or during DED, $V_{\mathrm{ph}}$ (symbols) is in good agreement with $V_{E \times B}$ (curves) in both the rotation direction and magnitude. With DED, the reversal of $E_{r}$ in the EZ/LZ area induces a change of $V_{\mathrm{ph}}$ from the $\omega_{*, e}$ to $\omega_{*, i}$ direction. The edge plasma polodial rotation is
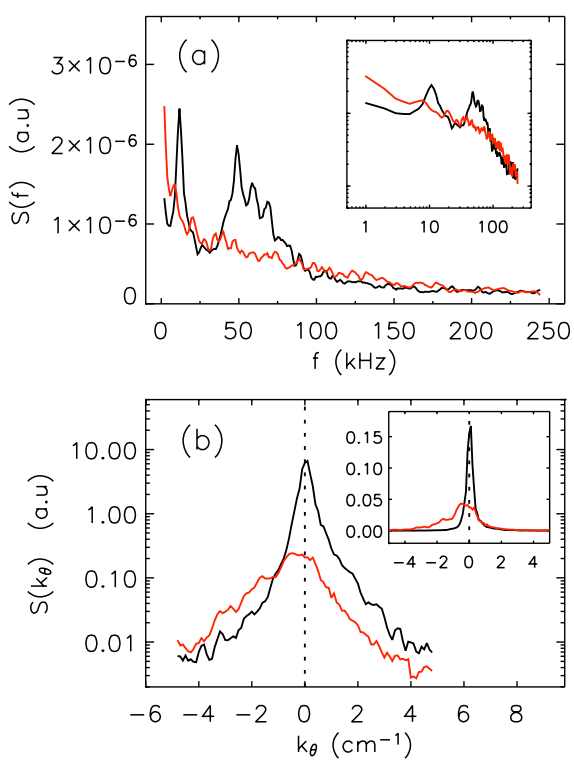

FIG. 4 (color online). (a) Linear plot of the frequency spectrum $S(f)$ of density fluctuations; (b) semilog plot of the poloidal wave-number spectrum $S\left(k_{\theta}\right)$ of potential fluctuations measured in the ergodic zone $(r=42.6 \mathrm{~cm})$ before (black curves) and during (red or gray curves) the static $m / n=6 / 2$ DED. The inset in (a) shows the log-log plot of $S(f)$ and in (b) the linear plot of $S\left(k_{\theta}\right)$ normalized by the total values before and during the DED. 

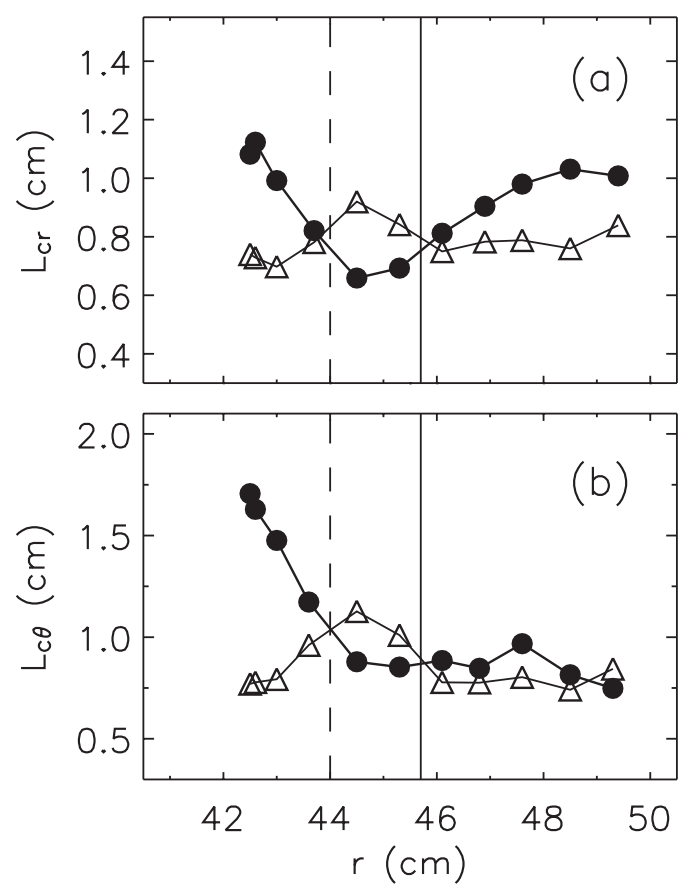

FIG. 5. Radial profiles of (a) the radial and (b) poloidal correlation lengths measured before (solid circles) and during (open triangles) the static $m / n=6 / 2$ DED. The vertical solid line indicates the limiter position and the dashed line separates the ergodic (left side) and laminar (right side) zones.

thus dominated by the $E_{r} \times B$ flow in both the Ohmic and the static DED period.

In conclusion, we report the first systematic measurements of the edge turbulence properties and of the turbulent flux under various static DED configurations on TEXTOR. Common features are observed, which unravel a controlling role by magnetic chaos on the edge turbulence in some intricate ways. In the ergodic zone, the local turbulent particle flux reverses direction from radially out-

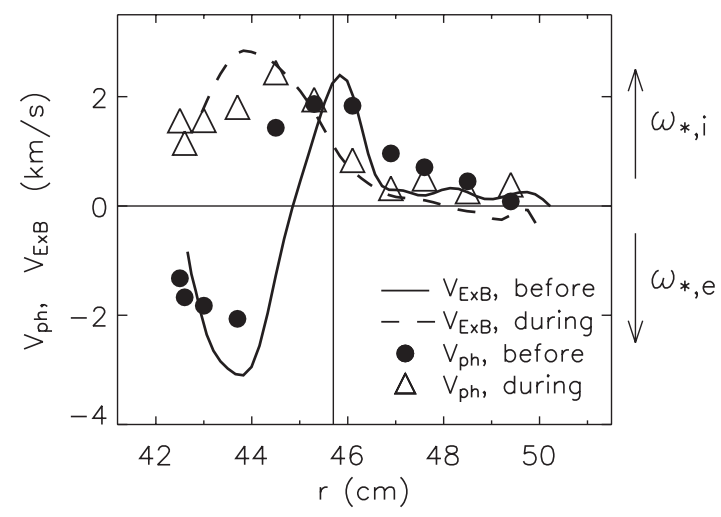

FIG. 6. Radial profiles of the poloidal phase velocity of fluctuations, $V_{\mathrm{ph}}$ (symbols), and the $E_{r} \times B$ drift velocity, $V_{E \times B}$ (curves), before and during the static $m / n=6 / 2$ DED. The $\omega_{*, i}$ $\left(\omega_{*, e}\right)$ denotes the ion (electron) diamagnetic drift direction. wards to inwards, which causes a steepening of the local pressure gradient but does not affect the global confinement. The turbulence itself is profoundly modified: energy redistribution occurs in frequency spectra, large scale turbulent eddies are suppressed, and the radial and poloidal correlation lengths decrease. In the same zone, the fluctuation poloidal phase velocity alters direction from electron to ion diamagnetic drift, consistent with the observed reversal of the $E_{r} \times B$ flow. In the immediate vicinity of the plasma edge where without the DED the customary flow shear enhancement around the last closed flux surface occurs, a laminar zone is created where the magnetic ergodization is no longer efficient and the turbulence is found to react to an observed reduced flow shear. The experimental results thus have significant implications for the understanding of electrostatic turbulence and the associated transport in the presence of magnetic ergodization.

The authors would like to thank Dr. P. Beyer, Dr. M. Tokar, and Dr. R. Singh for many useful discussions, B. Schweer, S. Musso, I. Lassiwe, and K. Bovert for implementation and maintenance of the fast reciprocating probe, Y. Liang for operational assistance, and R. Jaspers and M. Lehnen for support of this work.

*Partner in the Trilateral Euregio Cluster (TEC).

[1] S. C. McCool et al., Nucl. Fusion 29, 547 (1989).

[2] Ph. Ghendrih et al., Plasma Phys. Controlled Fusion 38, 1653 (1996); Ph. Ghendrich et al., Nucl. Fusion 42, 1221 (2002).

[3] T. E. Evans et al., Phys. Rev. Lett. 92, 235003 (2004).

[4] K. H. Finken et al., Plasma Phys. Controlled Fusion 46, B143 (2004).

[5] J. Payan et al., Nucl. Fusion 35, 1357 (1995).

[6] P. Devynck et al., Nucl. Fusion 42, 697 (2002).

[7] P. Beyer et al., Phys. Plasmas 5, 4271 (1998); P. Beyer et al., Plasma Phys. Controlled Fusion 44, 2167 (2002).

[8] D. Reiser et al., Phys. Plasmas 12, 122308 (2005).

[9] M.W. Jakubowski et al., Phys. Rev. Lett. 96, 035004 (2006).

[10] S. Chen and T. Sekiguchi, J. Appl. Phys. 36, 2363 (1965).

[11] P. C. Stangeby et al., Nucl. Fusion 30, 1225 (1990).

[12] J. M. Beall, Y. C. Kim, and E. J. Powers, J. Appl. Phys. 53, 3933 (1982).

[13] X. Z. Yang et al., Phys. Fluids B 3, 3448 (1991).

[14] A. Kramer-Flecken et al., Nucl. Fusion 46, S730 (2006).

[15] R. W. Harvey, M. G. McCoy, J. Y. Hsu, and A. A. Mirin, Phys. Rev. Lett. 47, 102 (1981).

[16] I. Kaganovich and V. Rozhansky, Phys. Plasmas 5, 3901 (1998).

[17] V. Rozhansky and M. Tendler, Phys. Fluids B 4, 1877 (1992).

[18] R. R. Weynants et al., in Proceedings of the 17th EPS Conference on Controlled Fusion and Plasma Heating, Amsterdam, 1990 (European Physical Society, Amsterdam, 1990), Vol. 14B, Pt. I, p. 287.

[19] A. J. Wootton, J. Nucl. Mater. 176, 77 (1990); Y. Xu et al., Phys. Plasmas 3, 1022 (1996). 